\title{
ESTILO DE VIDA E ADESÃO AO TRATAMENTO DE HIPERTENSÃO ARTERIAL SISTÊMICA EM HOMENS IDOSOS
}

\section{Lifestyle and adherence to treatment of systemic arterial hypertension in elderly men \\ Estilo de vida y adhesión al tratamiento de hipertensión arterial sistémica de hombres mayores}

\author{
Aline de Sousa Falcão \\ Universidade Federal do Piauí - UFPI - Teresina (PI) - Brasil \\ Maira Geny Carvalho e Silva \\ Universidade Federal do Piaú - UFPI - Teresina (PI) - Brasil
}

Adalberto Fortes Rodrigues Junior

Universidade Federal do Piaú - UFPI - Teresina (PI) - Brasil

Silmara da Rocha Moura

Universidade Federal do Piaú - UFPI - Teresina (PI) - Brasil

Flávia Raymme Soares e Silva

Universidade Federal do Piauí - UFPI - Teresina (PI) - Brasil

Antônia Sylea de Jesus Sousa

Universidade Federal do Piauí - UFPI - Teresina (PI) - Brasil

Erisonval Saraiva da Silva

Universidade Federal do Piauí - UFPI - Teresina (PI) - Brasil

Igho Leonardo do Nascimento Carvalho

Universidade Federal do Piauí - UFPI - Teresina (PI) - Brasil

\section{RESUMO}

Objetivo: Avaliar estilo de vida e adesão ao tratamento de hipertensão arterial sistêmica (HAS) em homens idosos. Métodos: Trata-se de estudo transversal, descritivo, com abordagem quantitativa, desenvolvido com 254 homens idosos, obedecendo aos seguintes critérios de inclusão: sexo masculino, idade igual ou superior a 60 anos e estar em acompanhamento da evolução da HAS na atenção primária. A coleta aconteceu por meio da aplicação de dois questionários: um semi-estruturado, contendo questões relacionadas a dados pessoais e familiares de condições de saúde e de estilo de vida, e o Questionário de Adesão ao Tratamento de Hipertensão Arterial Sistêmica (QTAHAS), entre outubro e dezembro de 2016. Resultados: A maioria dos homens idosos apresentou idade entre 70 e 79 anos (93; 36,5 \%), estado civil casado/união estável (174; $68,5 \%$ ), aposentado $(200 ; 78,7 \%)$. Houve predomínio dos hipertensos que deixam de tomar a medicação para HAS ao menos uma vez por ano, e comem praticamente sem gordura e sem doces e bebidas açucaradas (120; 47,2\%). O estilo de vida é marcado pela coexistência de grupos alimentares que favorecem ao controle e descontrole da adesão ao tratamento de hipertensão em homens idosos. Conclusão: Constatou-se a adesão parcial dos homens idosos avaliados, os quais utilizam alguns hábitos saudáveis como mecanismo compensatório para manter práticas que dificultam a adesão ao tratamento.

Descritores: Idoso; Estilo de vida; Hipertensão Arterial.

\section{ABSTRACT}

Objective: To assess the lifestyle and adherence to treatment of systemic arterial hypertension (SAH) in elderly men. Methods: This is a crosssectional and descriptive study with a quantitative approach, developed with 254 elderly men, meeting the following inclusion criteria: male, aged over 60 years, undergoing follow-up of SAH evolution in primary care. Data collection took place by means of two questionnaires: a semi-structured one, containing questions related to personal and family data on health and lifestyle conditions, and the Questionnaire on Adherence to the Treatment of Systemic Arterial Hypertension, between October and December 2016. Results: A majority of the elderly men were aged between 70 and 79 years (93; 36.5\%), marital status married/stable union (174; 68.5\%) and retired (200; 78.7\%). There was a predominance of hypertensive patients who stopped taking the antihypertensive medication at least once a year, and eat practically fat-free and

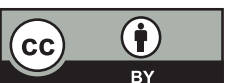

Este artigo está publicado em acesso aberto (Open Access) sob a licença Creative Commons, que permite uso, distribuição e reprodução em qualquer meio, sem restrições, desde que o trabalho seja corretamente citado. 
sugar-free foods, without consumption of sugary drinks (120; 47.2\%). Lifestyle is marked by the coexistence of food groups liable to improve or disturb the adherence to the treatment of hypertension in elderly subjects. Conclusion: Partial adherence was observed in the elderly men investigated, who adopt some healthy habits as a compensatory mechanism to maintain practices that hinder the adherence to treatment.

Descriptors: Elderly; Lifestyle; Hypertension.

\section{RESUMEN}

Objetivo: Evaluar el estilo de vida y la adhesión al tratamiento de la hipertensión arterial sistémica (HAS) en hombres mayores. Métodos: Se trata de un estudio transversal, descriptivo y de abordaje cuantitativo desarrollado con 254 hombres mayores obedeciendo a los siguientes criterios de inclusión: sexo masculino, edad de 60 años o más y estar en seguimiento de la evolución de la HAS en la atención primaria. La recogida de datos se dio a través de la aplicación de dos cuestionarios: uno semi-estructurado con preguntas relacionadas con los datos personales y familiares de las condiciones de salud y del estilo de vida y el Cuestionario de Adhesión al Tratamiento de Hipertensión Arterial Sistémica (CTAHAS) entre octubre y diciembre de 2016. Resultados: La mayoría de los hombres mayores presentó edad entre 70 y 79 años (93; 36,5\%), estado civil casado/unión estable (174; 68,5\%) y jubilado (200; 78,7\%). Hubo predominio de hipertensos que no toman la medicación para la HAS por lo menos una vez al año y comen alimentos casi sin grasa, dulces y bebidas con azúcar (120; 47,2\%). El estilo de vida está marcado por la coexistencia de grupos alimentarios que favorezcan el control y descontrol de la adhesión al tratamiento de la hipertensión en hombres mayores. Conclusión: Se constató una adhesión parcial de los hombres mayores evaluados los cuales utilizan algunos hábitos saludables como compensación del mantenimiento de prácticas que dificultan la adhesión al tratamiento.

Descriptores: Anciano; Estilo de vida; Hipertensión.

\section{INTRODUÇÃO}

O aumento da população idosa é um fenômeno mundial, correspondendo a 12 \% da população, o que representa 901 milhões de pessoas ${ }^{(1)}$. No Brasil, a proporção de crescimento da população idosa é semelhante, o que representa demandas clínicas e sociais e implicações para saúde pública(2).

Com o envelhecimento da população, há aumento da prevalência de doenças crônicas não transmissíveis (DCNT), com destaque para a hipertensão arterial sistêmica (HAS), devido à evolução assintomática por longo período de tempo e as dificuldades em seu controle, especialmente no caso de homens pela resistência na mudança do estilo de vida ${ }^{(3,4)}$.

A HAS é uma condição clínica multifatorial caracterizada por níveis elevados e sustentados de pressão arterial (PA) ( $\geq 140$ x $90 \mathrm{mmHg}$ ). Associa-se, frequentemente, às alterações funcionais e/ou estruturais dos órgãos-alvo (coração, encéfalo, rins e vasos sanguíneos) e às alterações metabólicas, com aumento do risco de eventos cardiovasculares fatais e não fatais, ocorrendo com maior prevalência em homens idosos ${ }^{(5)}$.

A HAS pode ser classificada de acordo com a medida casual, sendo classificada em estágio 1 (140-159/90-99 mmHg), 2 (160-179/ 100-109 mmHg) e 3 ( $\geq 180 / \geq 110 \mathrm{mmHg}$ ). Ainda existe a hipertensão do avental branco (valores anormais somente no consultório); a mascarada (valores normais somente no consultório) e a sistólica isolada (alteração exclusiva da pressão sistólica) ${ }^{(5)}$.

A prevalência média mundial estimada da HAS é de 26,4\%, atingindo 21,0\% nos EUA e Canadá, 33,5 a 39,7\% nos países europeus, 15 a 21,7\% nos países africanos e asiáticos, e cerca de 40\% na América Latina ${ }^{(6)}$.

Estudo epidemiológico realizado no Brasil apontou uma prevalência de HAS em 32,5\% (36 milhões) na população brasileira, com mais de $60 \%$ dos casos em idosos, sendo que, desse total, os homens representam maioria. Com isso, os homens idosos constituem a parte da população que apresenta maior prevalência de $\mathrm{HAS}^{(7)}$. Observa-se prevalência superior a Portugal, que apresenta prevalência na população de $17 \%$ e de homens idosos de $44,3 \%{ }^{(8)}$.

O serviço de saúde destinado ao acompanhamento integral e contínuo do paciente com HAS é a Estratégia Saúde da Família (ESF), cuja atuação visa assistir, diagnosticar e tratar a HAS, assegurando o controle da doença e a redução das complicações ${ }^{(9)}$.

O tratamento da HAS, que visa à redução da morbimortalidade cardiovascular, pode ter natureza não medicamentosa e/ou medicamentosa, na qual objetiva a redução gradual da PA para valores abaixo de $140 \mathrm{mmHg}$ para a pressão arterial sistólica (PAS) e $90 \mathrm{mmHg}$ para a pressão arterial diastólica (PAD) ${ }^{(5)}$.

O tratamento não medicamentoso, ou mudança no estilo de vida, é o recomendado para os idosos, devendo ser a primeira proposta terapêutica, pois é parte fundamental no controle da HAS, especialmente nos casos de hipertensão arterial leve, quando os níveis tensionais estão entre $140-159 / 90-99 \mathrm{mmHg}^{(10)}$.

Há eficácia comprovada dos hábitos relacionados ao estilo de vida saudável, inerente ao tratamento não medicamentoso, no controle dos níveis pressóricos e na diminuição das complicações. Esse tratamento envolve mudanças no estilo de vida, como prática de atividade física regular, redução do peso, controle lipídico, alimentação saudável, controle de estresse, cessação do tabagismo e do consumo do álcool, exigindo de seus portadores controle durante toda a vida ${ }^{(5,11)}$. 
A inatividade física tem sido considerada um dos maiores problemas de saúde pública por ser o mais prevalente dos fatores de risco para HAS. Por isso, a oferta de práticas de atividade física deve fazer parte dos trabalhos efetuados pela rede básica de saúde, já que o exercício físico regular reduz a PA e peso corporal ${ }^{(12)}$, muito útil para manter uma boa saúde cardiovascular e qualidade de $\operatorname{vida}^{(5)}$.

A adoção de hábitos relacionados ao estilo de vida saudável requer mudanças que não são facilmente realizadas, pois exigem disciplina e paciência para obter resultados ${ }^{(13)}$. Com isso, o tratamento não medicamentoso se constitui o item mais difícil de ser alcançado na adesão ao tratamento de HAS, pois requer um maior empenho por parte dos pacientes gerando um sério problema de saúde pública ${ }^{(14)}$.

Como benefícios da adesão ao tratamento não medicamentoso, evidenciam-se: o controle dos níveis pressóricos; a redução na incidência, ou retardamento, na ocorrência de complicações e a melhoria da qualidade de vida do idoso, sendo esta última a meta primordial das ações das equipes de saúde direcionadas a otimizar a adesão do idoso ao tratamento. Assim, a promoção da adesão ao tratamento de HAS é essencial para que os idosos alcancem qualidade de vida ${ }^{(15)}$, já que os idosos possuem maior facilidade em aderir ao tratamento medicamentoso do que realizar mudanças comportamentais e de estilo de vida ${ }^{(16)}$.

Logo, o trabalho foi impulsionado pela necessidade de se avaliar o grau de adesão dos idosos ao tratamento da HAS com ênfase no estilo de vida, uma vez que os estudos mostram existir baixa adesão às mudanças comportamentais e de estilo de vida, típicas do tratamento não medicamentoso ${ }^{(15,16)}$.

A adesão ao tratamento não medicamentoso é fundamental para a elaboração de estratégias que otimizem o envolvimento do idoso com as mudanças no estilo de vida, contribuindo no aprimoramento do conhecimento sobre o tratamento e a prevenção de complicações. Esta pesquisa, portanto, justifica-se pela pretensão em estimular os profissionais de saúde que atuam na ESF para a importância de orientarem e estimularem continuamente os idosos com HAS a adotarem um estilo de vida saudável, que contribua para a melhoria da qualidade de vida.

Este estudo tem como objetivo avaliar o estilo de vida e a adesão ao tratamento da hipertensão arterial sistêmica em homens idosos.

\section{MÉTODOS}

Trata-se de um estudo transversal, analítico, com abordagem quantitativa, realizado com 254 homens idosos hipertensos (erro amostral: 5\% e nível de confiança: 95\%) no município de Floriano, Piauí, Brasil, localizado a $240 \mathrm{~km}$ da capital do estado, Teresina, com população estimada de 57.690 habitantes.

A coleta de dados ocorreu no período de outubro a dezembro de 2016, sendo que os participantes foram submetidos aos seguintes critérios de inclusão: sexo masculino, idade igual ou superior a 60 anos e estar em acompanhamento da evolução da HAS por uma equipe de ESF. Excluíram-se os participantes com transtorno mental descompensado e com comorbidade em estágio avançado ou terminal.

Os homens idosos hipertensos foram pesquisados em um único momento em seus domicílios. A visita era previamente agendada pelo agente comunitário de saúde (ACS). A pesquisa aconteceu por meio da aplicação de dois questionários: um questionário semi-estruturado, contendo questões relacionadas a dados pessoais e familiares, de condições de saúde e de estilo de vida, e o Questionário de Adesão ao Tratamento de Hipertensão Arterial Sistêmica (QTAHAS) ${ }^{(17)}$.

Os dados pessoais e familiares abrangeram: a idade em anos completos; etnia autodeclarada; estado civil; escolaridade; renda individual e familiar em salários mínimos, considerando o valor $(\mathrm{R} \$ 880,00)$ no momento da coleta; desempenho de chefe da família relacionado com maior contribuição com renda familiar; ocupação ou profissão.

Os dados de condições de saúde abrangeram período de diagnóstico de HAS em anos completos, se possuía histórico familiar de $1^{\circ}$ grau de HAS, se possuía outra doença associada e se teve complicações decorrentes da HAS. A medida do índice de massa corporal (IMC) foi realizada por meio de balança digital Techline e fita métrica, seguindo classificação específica para idosos: baixo peso $\left(<22 \mathrm{Kg} / \mathrm{m}^{2}\right)$; peso adequado $\left(22-27 \mathrm{Kg} / \mathrm{m}^{2}\right)$ e sobrepeso $\left(>27 \mathrm{Kg} / \mathrm{m}^{2}\right)^{(1)}$. A medida da circunferência abdominal foi realizada com fita métrica, sendo que a interpretação foi de risco aumentado quando em homens $>94 \mathrm{~cm}$, sendo risco muito aumentado quando homens $>102 \mathrm{~cm}^{(18)}$.

Os dados sobre o estilo de vida abrangeram a prática de atividade física, tabagismo, uso de bebida alcoólica e dieta diária (incluem alimentos grelhados ou cozidos, frutas, legumes e verduras, carnes brancas e magras, carnes vermelhas ou gordas, temperados com pouco sal, fritos, enlatados ou industrializados, doces).

A avaliação da adesão ao tratamento de HAS ocorreu por meio da aplicação do instrumento QATHAS, validado ${ }^{(17)}$, composto por 12 (doze) questões e com coeficiente alfa $(\alpha)$ de Cronbach de 0,81. O QATHAS representa um instrumento hábil de avaliação da adesão ao tratamento da HAS e pode facilitar a detecção e aferição do cumprimento à terapêutica prescrita, além de viabilizar o estabelecimento de metas a serem alcançadas ${ }^{(19)}$.

A resposta é um valor do parâmetro $(\theta)$ estimado para o desempenho daquele participante. A escala pode variar entre $60 \mathrm{e}$ 110 , sendo que, quanto maior a pontuação, maior a adesão ao tratamento. 
Os dados foram armazenados e analisados no pacote estatístico Statistical Package for the Social Science - SPSS ${ }^{\circledR} 19.0$. O plano de análise compreendeu a estatística descritiva do perfil socioeconômico, condições de saúde, estilo de vida e classificação da adesão tratamento por meio de frequência absoluta e relativa, organizada em tabelas. Por fim, houve avaliação de associação da adesão ao tratamento de HAS com características do estilo de vida por meio do Teste Qui-quadrado $(\mathrm{p}<0,05)$ e razão de prevalência com intervalo de confiança de $95 \%$. Nessa estatística, considerou-se aderido o participante com QATHAS igual ou maior que 90, e não aderido o participante com classificação menor que 90.

O estudo recebeu aprovação do Comitê de Ética e Pesquisa (CEP), da Universidade Federal do Piauí (UFPI), respeitando os preceitos éticos da Resolução n ${ }^{\circ}$ 466/12 do Conselho Nacional de Saúde, com Parecer nº 1.668.881.

\section{RESULTADOS}

Considerando as variáveis socioeconômicas dos 254 idosos em tratamento de HAS, pôde-se observar predomínio da faixa etária de 70 a 79 anos $(93 ; 36,5 \%)$, da etnia parda (136; 53,5\%), com estado civil casado/união estável (174; 68,5\%) e escolaridade até o ensino fundamental $(128 ; 50,4 \%)$ (Tabela I).

A renda individual dos participantes foi de 1 a 3 SM $(218 ; 85,8 \%)$ e a renda familiar permaneceu a mesma $(205 ; 80,7 \%)$. Os participantes exercem o papel de chefe da família (204; 80,3\%), embora a maioria dos participantes seja aposentado (200; $78,7 \%$ ) (Tabela I).

Tabela I - Caracterização socioeconômica dos idosos em tratamento de hipertensão arterial sistêmica. Floriano, Piauí, Brasil, 2016.

\begin{tabular}{|c|c|c|}
\hline Variáveis & $\begin{array}{l}\text { Frequência Absoluta } \\
\text { (n) }\end{array}$ & $\begin{array}{c}\begin{array}{c}\text { Frequência Relativa } \\
(\%)\end{array} \\
\end{array}$ \\
\hline \multicolumn{3}{|l|}{ Faixa etária } \\
\hline 60 a 69 anos & 89 & 35 \\
\hline 70 a 79 anos & 93 & 36,5 \\
\hline 80 a 89 anos & 59 & 23,3 \\
\hline Acima de 90 anos & 13 & 5,2 \\
\hline \multicolumn{3}{|l|}{ Etnia } \\
\hline Branco & 40 & 15,8 \\
\hline Pardo & 136 & 53,5 \\
\hline Negro & 72 & 28,3 \\
\hline Amarelo & 6 & 2,4 \\
\hline \multicolumn{3}{|l|}{ Estado Civil } \\
\hline Solteiro & 16 & 6,3 \\
\hline Casado/União estável & 174 & 68,5 \\
\hline Viúvo & 46 & 18,1 \\
\hline Divorciado & 18 & 7,1 \\
\hline \multicolumn{3}{|l|}{ Escolaridade } \\
\hline Não alfabetizado & 90 & 35,4 \\
\hline Ensino fundamental & 128 & 50,4 \\
\hline Ensino médio & 26 & 10,2 \\
\hline Ensino superior completo & 10 & 4,0 \\
\hline \multicolumn{3}{|l|}{ Renda individual } \\
\hline Menos de $1 \mathrm{SM}$ & 14 & 5,5 \\
\hline $1-3 \mathrm{SM}$ & 218 & 85,8 \\
\hline $3-6 \mathrm{SM}$ & 22 & 8,7 \\
\hline \multicolumn{3}{|l|}{ Papel de chefe da família } \\
\hline Sim & 204 & 80,3 \\
\hline Não & 50 & 19,7 \\
\hline \multicolumn{3}{|l|}{ Ocupação } \\
\hline Exerce atividade de trabalho & 54 & 21,3 \\
\hline Aposentando & 200 & 78,7 \\
\hline
\end{tabular}

n: número de indivíduos; SM: salários mínimos.

As condições de saúde dos participantes são caracterizadas pelo período de diagnóstico de até 5 anos $(82 ; 32,3 \%)$, possuem prescrição medicamentosa $(242 ; 95,3 \%)$ e familiar com HAS $(158 ; 62,2 \%)$. A maioria dos participantes possui peso adequado 
(119; 46,9\%), mas a circunferência abdominal aumentada (164; 65\%). Quanto aos níveis pressóricos, a maioria dos participantes apresenta hipertensão estágio 1 (86; 36,9\%) (Tabela II).

Tabela II - Caracterização da condição de saúde dos idosos com hipertensão arterial sistêmica (HAS). Floriano, Piaú, Brasil, 2016.

\begin{tabular}{lcc}
\hline Variáveis & $\begin{array}{c}\text { Frequência Absoluta } \\
\text { (n) }\end{array}$ & $\begin{array}{c}\text { Frequência Relativa } \\
\text { (\%) }\end{array}$ \\
\hline Período de diagnóstico da HAS & 82 & 32,3 \\
Até 05 anos & 76 & 29,9 \\
$05-10$ & 37 & 14,6 \\
$10-15$ & 59 & 23,2 \\
Acima de 15 anos & & \\
Prescrição medicamentosa & 242 & 95,3 \\
Sim & 12 & 4,7 \\
Não & & \\
Familiar com HAS & 158 & 62,2 \\
Sim & 96 & 37,8 \\
Não & & \\
Possui outra doença & 129 & 50,8 \\
Sim & 125 & 49,2 \\
Não & & 16,5 \\
Classificação do IMC & 42 & 46,9 \\
Baixo peso idoso (<22) & 119 & 36,6 \\
Peso adequado idoso (22 - 27) & 93 & 35,0 \\
Sobrepeso idoso (> 27) & & 65,0 \\
Circunferência abdominal & 89 & \\
Adequada (<94 cm) & 164 & 29,1 \\
Aumentada (>94cm) & & 20,5 \\
Pressão Arterial & 74 & 33,9 \\
Normal & 52 & 6,7 \\
Pré-Hipertensão & 86 & \\
Hipertensão estágio 1 & 25 & \\
Hipertensão estágio 2 & 17 & \\
Hipertensão estágio 3 & & \\
\hline
\end{tabular}

HAS: hipertensão arterial sistêmica; IMC: índice de massa corpórea; $\mathrm{cm}=$ centímetros.

A adesão ao tratamento da HAS dos participantes obteve todos os níveis de classificação, mas com predomínio do nível em que os hipertensos deixam de tomar a medicação para HAS ao menos uma vez por ano, e comem praticamente sem gordura e sem doces e bebidas açucaradas $(120 ; 47,2 \%)$. Outro nível representativo foi o em que os hipertensos deixam de tomar a medicação nos horários estabelecidos ao menos uma vez por mês; reduziram à metade o sal, a gordura, e os doces e bebidas com açúcar (83; 32,7\%) (Tabela III).

A adesão dos participantes ao tratamento não medicamentoso é caracterizada pela equivalente frequência entre prática e não prática de exercícios físicos, e absenteísmo de fumo (204; 90,7\%) e de bebida alcoólica (180; 80\%). Quanto aos alimentos saudáveis, os participantes consomem alimentos grelhados (210; 93,3\%); frutas, legumes e verduras (209; 92,9\%); carnes brancas e magras (199; 88,4\%); alimentos hipossódicos $(212 ; 94,2 \%)$. Quanto aos alimentos não indicados, os participantes relataram não consumir frituras $(128 ; 56,9 \%)$; enlatados ou industrializados $(182 ; 80,9 \%)$; doces $(138 ; 61,3 \%)$, mas consomem carnes vermelhas ou gordas $(168 ; 74,7 \%)$ (Tabela IV).

Constatou-se relação significativa entre adesão do tratamento da HAS ao absenteísmo de fumo; consumo de frutas, verduras e legumes; consumo de carnes brancas e magras, constituindo fatores de proteção. Ainda apresenta relação com alimentos fritos, industrializadas, ou enlatados, e doces, constituindo fatores de exposição (Tabela IV).

Identificaram-se hábitos e alimentos que reduzem a prevalência da adesão, tais como: alimentos enlatados reduzem em 67\%; os alimentos fritos e doces reduzem em $72 \%$; e o hábito de fumar reduz em $81 \%$ a adesão ao tratamento de HAS. Também foram identificados alimentos que aumentam mais que o triplo a prevalência da adesão, tais como carnes brancas e magras, alimentos hipossódicos e frutas, legumes e verduras. 
Tabela III - Classificação da adesão ao tratamento de hipertensão arterial sistêmica segundo Questionário de Adesão ao Tratamento de Hipertensão Arterial Sistêmica (QTAHAS). Floriano, Piauí, Brasil, 2016.

\begin{tabular}{|c|c|c|c|}
\hline Descrição & $\begin{array}{c}\text { Nível } \\
\text { da escala }\end{array}$ & $\begin{array}{l}\text { Frequência } \\
\text { Absoluta (n) }\end{array}$ & $\begin{array}{c}\text { Frequência } \\
\text { Relativa }(\%)\end{array}$ \\
\hline $\begin{array}{l}\text { Neste nível, os hipertensos não tomam o anti-hipertensivo ao menos uma } \\
\text { vez por semana. } E \text { também não o tomam, ao menos uma vez por semana, na } \\
\text { dose prescrita. }\end{array}$ & $<70$ & 1 & 0,4 \\
\hline $\begin{array}{l}\text { Os hipertensos posicionados neste nível deixam de tomar a medicação para } \\
\text { hipertensão nos horários estabelecidos ao menos uma vez por semana e } \\
\text { comparecem às consultas agendadas. }\end{array}$ & $70-79,9$ & 4 & 1,6 \\
\hline $\begin{array}{l}\text { Ao atingirem este nível, os hipertensos deixam de tomar a medicação conforme a } \\
\text { dose prescrita ao menos uma vez por mês, fazem uso da medicação independente } \\
\text { de sentir algum sintoma, seguem o tratamento medicamentoso rotineiramente } \\
\text { e reduziram a terça parte do sal, da gordura, e de doces e bebidas com açúcar. }\end{array}$ & $80-89,9$ & 24 & 9,4 \\
\hline $\begin{array}{l}\text { Os hipertensos localizados neste nível deixam de tomar a medicação, nos } \\
\text { horários estabelecidos ao menos uma vez por mês; reduziram à metade o sal, } \\
\text { gordura, e doces e bebidas com açúcar. }\end{array}$ & $90-99,9$ & 83 & 32,7 \\
\hline $\begin{array}{l}\text { Neste nível, os hipertensos deixam de tomar a medicação para hipertensão } \\
\text { ao menos uma vez por ano, e comem praticamente sem gordura e sem doces } \\
\text { e bebidas com açúcar. }\end{array}$ & $100-109,9$ & 120 & 47,2 \\
\hline $\begin{array}{l}\text { A partir deste nível, os hipertensos não deixam de tomar a medicação para } \\
\text { hipertensão, comem praticamente sem sal e seguem o tratamento não } \\
\text { medicamentoso rotineiramente. }\end{array}$ & $>110$ & 22 & 8,7 \\
\hline
\end{tabular}

Tabela IV - Avaliação da relação entre adesão ao tratamento de hipertensão arterial sistêmica e estilo de vida. Floriano, Piauí, Brasil, 2016.

\begin{tabular}{|c|c|c|c|c|}
\hline Variáveis & $\begin{array}{c}\text { Adesão } \\
\text { n (\%) }\end{array}$ & $\begin{array}{c}\text { Não adesão } \\
\text { n (\%) }\end{array}$ & Valor $\mathbf{p}$ & RP (IC95\%) \\
\hline \multicolumn{5}{|c|}{ Pratica exercícios físicos } \\
\hline $\operatorname{Sim}$ & $109(48,4)$ & $12(41,4)$ & \multirow[b]{2}{*}{0,473} & 1,33 \\
\hline Não & $116(51,6)$ & $17(58,6)$ & & $(0,60-2,91)$ \\
\hline \multicolumn{5}{|l|}{ Fumo } \\
\hline Sim & $21(9,3)$ & $10(34,5)$ & \multirow{2}{*}{$0,000 *$} & 0,19 \\
\hline Não & $204(90,7)$ & $19(65,5)$ & & $(0,08-0,47)$ \\
\hline \multicolumn{5}{|c|}{ Uso de bebida alcoólica } \\
\hline Sim & $45(20,0)$ & $9(31,0)$ & \multirow{2}{*}{0,172} & \\
\hline Não & $180(80,0)$ & $20(69,0)$ & & $(0,23-1,30)$ \\
\hline \multicolumn{5}{|c|}{ Alimentos grelhados ou cozidos } \\
\hline Sim & $210(93,3)$ & $26(89,7)$ & \multirow{2}{*}{0,468} & \\
\hline Não & $15(6,7)$ & $3(10,3)$ & & $(0,43-5,95)$ \\
\hline \multicolumn{5}{|c|}{ Frutas, legumes e verduras } \\
\hline Sim & $209(92,9)$ & $17(58,6)$ & \multirow{2}{*}{$0,000 *$} & \\
\hline Não & $16(7,1)$ & $12(41,4)$ & & $(3,76-22,6)$ \\
\hline \multicolumn{5}{|c|}{ Carnes brancas e magras } \\
\hline Sim & $199(88,4)$ & $20(69,0)$ & \multirow{2}{*}{$0,004 *$} & 3,44 \\
\hline Não & $26(11,6)$ & $9(31,0)$ & & $(1,41-8,35)$ \\
\hline \multicolumn{5}{|c|}{ Alimentos hipossódicos } \\
\hline Sim & $212(94,2)$ & $23(79,3)$ & \multirow{2}{*}{$0,004^{*}$} & \\
\hline Não & $13(5,8)$ & $6(20,7)$ & & $(1,47-12,2)$ \\
\hline \multicolumn{5}{|c|}{ Alimentos fritos } \\
\hline Sim & $97(43,1)$ & $21(72,4)$ & \multirow{2}{*}{0,003} & 0,28 \\
\hline Não & $128(56,9)$ & $8(27,6)$ & & $(0,12-0,67)$ \\
\hline \multicolumn{5}{|c|}{ Enlatados ou industrializados } \\
\hline Sim & $43(19,1)$ & $12(41,4)$ & \multirow[b]{2}{*}{$0,006^{*}$} & \\
\hline Não & $182(80,9)$ & $17(58,6)$ & & $(0,14-0,75)$ \\
\hline \multicolumn{5}{|l|}{ Doces } \\
\hline Sim & $87(38,7)$ & $20(69,0)$ & \multirow{2}{*}{$0,002 *$} & \\
\hline Não & $138(61,3)$ & $9(31,0)$ & & $(0,12-0,65)$ \\
\hline \multicolumn{5}{|c|}{ Carnes vermelhas ou gordas } \\
\hline Sim & $168(74,7)$ & $25(86,2)$ & \multirow{2}{*}{0,171} & 0,47 \\
\hline Não & $57(25,3)$ & $4(13,8)$ & & $(0,15-1,41)$ \\
\hline
\end{tabular}

Qui-quadrado (p); Razão de prevalência (RP); Intervalo de Confiança (IC) 


\section{DISCUSSÃO}

O presente estudo evidenciou o aumento da ocorrência da HAS associado à idade, o que coincide com outros estudos ${ }^{(20,21)}$. A associação direta e linear entre envelhecimento e prevalência de HAS, deve-se ao envelhecimento vascular caracterizado por alterações na microarquitetura da parede dos vasos, com consequente enrijecimento arterial ${ }^{(11)}$.

A maioria dos participantes da atual investigação era pardos, o que coincide com estudo realizado em João Pessoa, Brasil, onde $48 \%$ dos hipertensos eram pardos ${ }^{(22)}$. Nesse sentido, sabe-se que a HAS é duas vezes mais prevalente em indivíduos de cor não branca ${ }^{(5)}$.

No presente estudo, os participantes eram casados ou viviam em união estável, corroborando o estudo realizado em São Paulo, em que $63 \%$ dos participantes eram casados ${ }^{(23)}$. O estado civil casado ou união estável pode influenciar na maior adesão ao tratamento por causa do incentivo do parceiro (a) em seguir as recomendações de saúde ${ }^{(24)}$.

Em relação à baixa escolaridade dos participantes do estudo em questão, sabe-se que influencia na dificuldade de controle eficaz da pressão arterial e na maior prevalência de HAS. Logo, o grau de instrução é considerado um dos fatores determinantes da adesão terapêutica ${ }^{(25)}$. A associação entre controle da HAS e menor escolaridade pode refletir a dificuldade para o conhecimento da doença e seus fatores de risco, bem como adesão a medidas de controle ${ }^{(26)}$.

A renda entre 1 e 3 salários foi predominante entre os participantes da atual pesquisa, que, por sua vez, tem como ocupação serem aposentados/pensionistas. Tais dados corroboram o achado do estudo realizado em João Pessoa, Brasil, em que $84 \%$ dos participantes apresentavam renda mensal de 1 a 3 salários $^{(22)}$. O baixo nível socioeconômico é identificado como um fator que dificulta o controle efetivo da adesão ao tratamento de $\operatorname{HAS}^{(17,20)}$.

A HAS é uma doença silenciosa e de longo curso assintomático, sendo o diagnóstico recente favorece ao menor índice de abandono. No presente estudo, o período de diagnóstico da HAS mais frequente foi de até 5 anos, convergindo para mesmo achado um estudo na cidade de São Paulo, Brasil, em que 51,6 \% dos participantes possuíam diagnóstico entre 1 e 5 anos, PA sob controle ${ }^{(27)}$.

Os participantes da presente pesquisa possuíam prescrição medicamentosa, o que sugere ações na atenção primária e na melhoria do acesso aos serviços de saúde ${ }^{(28)}$. Sabe-se que a decisão do momento de início da medicação anti-hipertensiva deve ser considerada avaliando a preferência da pessoa, o seu grau de motivação para mudança de estilo de vida, os níveis pressóricos e o risco cardiovascular ${ }^{(10)}$.

No atual estudo, os participantes tinham histórico familiar de HAS, o que coincide com estudo que encontrou associação entre a história familiar de HAS em $86,7 \%$ dos idosos da amostra ${ }^{(3)}$. Com isso, ratifica-se a influência do fator hereditário no surgimento das doenças cardiovasculares (DCV).

O desenvolvimento da HAS se constitui um fator de risco para outras doenças, especialmente no sistema endócrino, tal como a diabetes mellitus (DM), o que justifica a existência de outras doenças entre os participantes ${ }^{(11)}$. A HAS constitui-se ainda um importante fator de risco para complicações cardíacas e cerebrovasculares, com repercussões qualidade de vida dos indivíduos implicando negativamente na avaliação geral de qualidade de vida ${ }^{(29,30)}$.

O presente estudo evidenciou que a maioria dos idosos encontra-se com índice de massa corporal (IMC) adequado, entretanto a circunferência abdominal (CA) apresenta-se aumentada. A CA mostra-se um importante indicador antropométrico, devido sua associação significativa com a $\mathrm{HAS}^{(31)}$. A composição corporal do homem idoso apresenta tendência acentuada de deposição de gordura da região abdominal, o que caracteriza essa alteração. O aumento da gordura visceral é considerado um fator de risco para $\operatorname{HAS}^{(11)}$.

No momento da coleta do atual estudo, verificou-se que a maioria dos participantes apresentava descontrole pressórico, achado semelhante ao estudo no qual $87,9 \%$ dos pacientes apresentou níveis pressóricos acima dos limites de normalidade num estudo realizado no Rio de Janeiro ${ }^{(32)}$.

A adesão parcial dos participantes da atual investigação demonstra a complexidade envolvida no seguimento das condutas terapêuticas inerentes ao tratamento de HAS. Em estudo desenvolvido no município de Picos, estado do Piauí, com 118 idosos, foi observado o mesmo nível de adesão após atividade de educação em saúde ${ }^{(33)}$.

$\mathrm{O}$ atual estudo mostrou que os participantes não realizam atividade física, coincidindo com o achado de uma pesquisa em que 65,9\% dos participantes não praticavam atividade física no Piauí ${ }^{(34)}$, alegando falta de motivação advinda da idade e dificuldade em mudar hábitos de vida. O sedentarismo é considerado o fator de risco mais prevalente para $\operatorname{HAS}^{(22)}$.

O efeito protetor da atividade física vai além da redução da PA, estando associado à redução dos fatores de risco cardiovasculares e à menor morbimortalidade quando comparadas pessoas ativas com indivíduos de menor aptidão física, o que explica a sua recomendação na prevenção primária e no tratamento da hipertensão ${ }^{(35)}$.

O absenteísmo do tabagismo e do álcool são dois hábitos indicados para adesão ao tratamento de HAS e controle da PA. $\mathrm{O}$ abandono do tabagismo é condição essencial para reduzir o risco de DCV, obrigatória na abordagem ao portador de HAS, enquanto a redução ou abandono do consumo do álcool reduz a $\mathrm{PA}^{(11)}$. 
Observou-se no estudo em questão a relação da adesão ao tratamento de HAS e o consumo de um grupo de alimentos saudáveis (grelhados, cozidos, frutas, legumes, verduras, carnes brancas e magras, hipossódicos) e com alimentos que não são indicados no tratamento não medicamentos de HAS (fritos, enlatados, doces) ${ }^{(33)}$. Tal achado demonstra um mecanismo compensatório dos participantes, ingerindo alimentos saudáveis e não indicados, e sugere que os participantes busquem o controle da HAS, mas não abandonam o consumo dos alimentos de exposição.

Achados semelhantes foram encontrados no estudo com 385 hipertensos em que as mudanças na dieta foram relatadas por 266 (69,1 \%). Destes, a grande maioria $(99,6 \%$ ) alegou redução do consumo de alimentos de risco, sendo a restrição do consumo de sal $(84,2 \%)$, gorduras $(36,2 \%)$ e doces $(26,0 \%)$ os mais referidos ${ }^{(36)}$. De maneira complementar, um estudo mostrou que $85,2 \%$ dos participantes mantêm pelo menos um hábito de vida não saudável ${ }^{(37)}$ e outro estudo relatou a dificuldade de adesão ao tratamento não medicamentoso em longo prazo $^{(38)}$.

Vale ressaltar que os achados evidenciaram que os idosos avaliados na presente pesquisa, mesmo possuindo uma baixa condição socioeconômica, conseguem consumir alimentos saudáveis. No entanto, a ausência de um padrão-ouro para avaliar consumo alimentar, sobretudo em população idosa, é uma importante limitação ${ }^{(39)}$.

Os profissionais de saúde responsáveis pelo acompanhamento dos idosos com HAS podem usar a escuta no planejamento ações resolutivas; educação em saúde; orientação sobre hábitos de vida saudáveis de forma clara, com o objetivo do autocuidado; e práticas humanizadas promotoras da empatia, pois facilitam a adesão, o controle pressórico e propiciam o sucesso do tratamento ${ }^{(40)}$.

Assim, os profissionais de saúde podem contribuir de forma significativa para a melhoria nas condições de saúde e qualidade de vida do portador de HAS. Os profissionais de saúde deveriam estabelecer uma relação dialogada e realizar ações educativas com os idosos para demonstrar a importância da mudança integral no estilo de vida, preservando seu empoderamento. Tais estratégias visam o controle da pressão arterial e redução de riscos de complicações, subsidiando uma assistência qualificada e integral dos idosos com HAS.

\section{CONCLUSÃO}

O estudo da adesão ao tratamento de hipertensão arterial sistêmica, com ênfase no estilo de vida em homens idosos, constatou a adesão parcial dos homens idosos avaliados, que utilizam alguns hábitos saudáveis como mecanismo compensatório para manter práticas que dificultam a adesão ao tratamento.

\section{CONTRIBUIÇÕES}

Aline de Sousa Falcão elaboração e delineamento do estudo; redação e/ou revisão do manuscrito. Maira Geny Carvalho e Silva aquisição, análise e interpretação de dados. Adalberto Fortes Rodrigues Junior aquisição, análise e interpretação de dados. Silmara da Rocha Moura aquisição, análise e interpretação de dados. Flávia Raymme Soares e Silva aquisição, análise e interpretação de dados. Antônia Sylea de Jesus Sousa redação e/ou revisão do manuscrito. Erisonval Saraiva da Silva redação e/ou revisão do manuscrito Igho Leonardo do Nascimento Carvalho elaboração e delineamento do estudo; redação e/ou revisão do manuscrito.

\section{REFERÊNCIAS}

1. World Health Organization. Adherence to long-term therapies: policy for action. Genebra: WHO; 2001 [acesso em 2016 Out 20]. Disponível em: http://www.who.int/chronic_conditions/en/adherencerep.pdf

2. Instituto Brasileiro de Geografia e Estatística. Estimativas da população residente no Brasil e unidades da Federação com data de referência em $1^{\circ}$ de julho de 2013. Rio de Janeiro: IBGE; 2013 [acesso em 2017 Out 15]. Disponível em: https:// ww2.ibge.gov.br/home/estatistica/populacao/estimativa2015/estimativa_dou.shtm

3. Nunes TM, Martins AM, Manoel AL, Trevisol DJ, Trevisol FS, Cavalcante RASQ, et al. Hipertensão arterial sistêmica em idosos do município de Tubarão, SC - Brasil: estudo populacional. Int J Cardiovasc Sci. 2015;28(5):370-6.

4. Freitas JGA, Nielson SEO, Porto CC. Adesão ao tratamento farmacológico em idosos hipertensos: uma revisão integrativa da literatura. Rev Soc Bras Clin Med. 2015;13(1):75-84.

5. Sociedade Brasileira de Cardiologia. VI Diretrizes Brasileiras de Hipertensão. Arq Bras Cardiol. 2010;95(1 Supl 1):1-51.

6. Cipullo JP, Martin JFV, Ciorlia LAS, Godoy MRP, Cação JC, Loureiro AAC, et al. Prevalência e fatores de risco par hipertensão em uma população urbana brasileira. Arq Bras Cardiol. 2010;94(4):519-26.

7. Scala LC, Magalhães LB, Machado A. Epidemiologia da hipertensão arterial sistêmica. In: Moreira SM, Paola AV. Sociedade Brasileira de Cardiologia: livro texto da Sociedade Brasileira de Cardiologia. $2^{\mathrm{a}}$ ed. São Paulo: Manole; 2015. p. 780-5. 
8. Ministério da Saúde (POR), Uva MS, Victorino P, Roquette R, Machado A, Dias CM. Prevalência e incidência da hipertensão arterial na população portuguesa. Lisboa: Ministério da Saúde; 2014.

9. Coutinho FHP, Sousa IMC. Percepção dos indivíduos com hipertensão arterial sobre sua doença e adesão ao tratamento medicamentoso na estratégia de saúde da família. Rev Baiana Saúde Pública. 2011;35(2):397-411.

10. Ministério da Saúde (BR), Secretaria de Atenção à Saúde, Departamento de Atenção Básica. Estratégias para o cuidado da pessoa com doença crônica: hipertensão arterial sistêmica. Brasília: Ministério da Saúde; 2013 [acesso em 2017 Set 10 ]. (Cadernos da Atenção Básica, n. 37). Disponível em: http://189.28.128.100/dab/docs/portaldab/publicacoes/caderno_37. pdf

11. Sociedade Brasileira de Hipertensão, Departamento de Hipertensão Arterial. Hipertensão ou Pressão alta. São Paulo: SBH; 2016 [acesso em 2016 Set 22]. Disponível em: www.sbh.org.br/geral/faq.asp

12. Ministério da Saúde (BR), Departamento de Atenção Básica. Política Nacional de Atenção Básica. Brasília: Ministério da Saúde; 2012 [acesso em 2016 Set 22]. Disponível em: http://dab.saude.gov.br/portaldab/smp_como_funciona.php

13. Gravina CF, Grespan SM, Borges JL. Tratamento não-medicamentoso da hipertensão no idoso. Rev Bras Hipertens. 2007;14(1):33-6.

14. Contiero AP, Pozati MPS, Challouts RI, Carreira L, Marcon SS. Idoso com hipertensão arterial: dificuldades de acompanhamento na Estratégia Saúde da Família. Rev Gaúch Enferm. 2009;30(1):62-70.

15. Baldissera VDA, Carvalho MDB, Pelloso SM. Adesão ao tratamento não-farmacológico entre hipertensos de um centro de saúde escola. Rev Gaúch Enferm. 2009;30(1):27-32.

16. Soares MM, Silva LOL, Dias CA, Rodrigues SM, Machado CJ. Adesão do idoso ao tratamento da hipertensão arterial sistêmica: revisão integrativa. Cogitare Enferm. 2012;17(1):144-50.

17. Rodrigues MTP. Adesão ao tratamento da hipertensão arterial sistêmica: desenvolvimento de um instrumento avaliativo com base na Teoria da Resposta ao Item (TRI) [tese]. Fortaleza: Universidade Estadual do Ceará, Universidade Federal do Ceará, Universidade de Fortaleza; 2012.

18. Ministério da Saúde (BR), Secretaria de Atenção à Saúde, Departamento de Ações Programáticas Estratégicas. Caderneta de saúde da pessoa idosa. 4a ed. Brasília: Ministério da Saúde; 2017 [acesso em 2017 Out 22]. Disponível em: http:// portalarquivos2.saude.gov.br/images/pdf/2017/setembro/27/CADERNETA-PESSOA-IDOSA-2017-Capa-miolo.pdf

19. QATHAS. Questionário de adesão ao tratamento da hipertensão arterial sistêmica [acesso em 2016 Out 16]. Disponível em: http://www.qathas.com.br/

20. Machado CR. Prevalência de hipertensão arterial e fatores associados, Cambé - PR [monografia]. Londrina: Centro Universitário Filadélfia; 2012.

21. Picon RV, Fuchs FD, Moreira LB, Fuchs SC. Prevalence of hypertension among elderly persons in urban Brazil: a systematic review with meta-analysis. Am J Hypertens. 2013;26(4):541-8.

22. Dourado CS, Macedo-Costa KNF, Oliveira JS, Leabedal ODCP, Silva GRF. Adesão ao tratamento de idosos com hipertensão em uma unidade básica de saúde de João Pessoa, Estado da Paraíba. Acta Sci. Health Sci. 2011;33(1):9-17.

23. Bastos-Barbosa RG, Ferriolli E, Moriguti, JC, Nogueira CB, Nobre F, Ueta J, et al. Adesão ao tratamento e controle da pressão arterial em idosos hipertensos. Arq Bras Cardiol, 2012;99(1):636-41.

24. Kim MM, Howard DL, Kaufman JS, Holmes D. Predicting medication use in an elderly hypertensive sample: revisiting the Established Populations for Epidemiologic Studies of the Elderly Study. J Natl Med Assoc. 2008;100(12):1386-93.

25. Mendes GS, Moraes CF, Gomes L. Prevalência de hipertensão arterial sistêmica em idosos no Brasil entre 2006 e 2010. Rev Bras Med Fam Comunidade. 2014;9(32):273-8.

26. Menezes TN, Oliveira ECT, Fischer MATS, Esteves GH. Prevalência e controle da hipertensão arterial em idosos: um estudo populacional. Rev Port Saúde Pública. 2016;34(2):117-24.

27. Pierin AMG, Marroni SN, Taveira LAF, Bensenor IJM. Controle da hipertensão arterial e fatores associados na atenção primária em unidades básicas de saúde localizadas na região oeste da cidade de São Paulo. Ciênc Saúde Colet. 2011;16(Supl 1):1389-400.

28. Ferreira RA, Barreto SM, Giatti L. Hipertensão arterial referida e utilização de medicamentos de uso contínuo no Brasil: um estudo de base populacional. Cad Saúde Púlica. 2014;30(4):815-26. 
29. World Health Organization. Global atlas on cardiovascular disease prevention and control. Mendis S, Puska P, Norrving B, editors. Geneva: WHO; 2011.

30. Alves-Silva LS. Hipertensão arterial sistêmica e morbidade cardiovascular: foco na epidemiologia no Brasil. Rev Bras Hipertens. 2014;17(3-4):163-8.

31. Radovanovic CAT, Santos LA, Carvalho MDB, Marcon SS. Hipertensão arterial e outros fatores de risco associados às doenças cardiovasculares em adultos. Rev Latinoam. Enferm. 2014;22(4):547-53.

32. Bonadiman RL, Bonadiman SL, Silva DA. Avaliação da adesão ao tratamento medicamentoso e não medicamentoso de pacientes hipertensos atendidos no PSF Guaritá, Itaperuna-RJ. Acta Biomed Bras. 2012;3(1):73-84.

33. Machado ALG. Efeito do circulo de cultura na adesão ao tratamento e no letramento em saúde de idosos hipertensos [tese]. Fortaleza: Universidade Federal do Ceará; 2015.

34. Vieira CPB, Nascimento JJ, Barros SS, Luz MHBA, Valle ARMC. Prevalência referida, fatores de risco e controle da hipertensão arterial em idosos. Ciênc Cuid Saúde. 2016;15(3):413-20.

35. Nogueira IC, Santos ZMSA, Mont'Alverne DGB, Martins ABT, Magalhães CBA. Efeitos do exercício físico no controle da hipertensão arterial em idosos: uma revisão sistemática. Rev Bras Geriatr Gerontol. 2012;15(3):587-601.

36. Girotto E, Andrade SM, Cabrera MAS, Matsuo T. Adesão ao tratamento farmacológico e não farmacológico e fatores associados na atenção primária da hipertensão arterial. Ciênc Saúde Coletiva 2013;18(6):1763-72.

37. Silva LFRS, Marino JMR, Guidoni CM, Girotto E. Fatores associados à adesão ao tratamento anti-hipertensivo por idosos na atenção primária. Rev Ciênc Farm Básica Apl. 2014;35(2):271-8.

38. Pereira IMO. Proposta de intervenção interdisciplinar para a adesão dos pacientes ao tratamento da hipertensão arterial sistêmica. Liph Science. 2015;2(2):21-40.

39. Souza JD, Martins MV, Franco FS, Martinho KO, Tinôco AL. Padrão alimentar de idosos: caracterização e associação com aspectos socioeconômicos. Rev Bras Geriatr Gerontol. 2016;19(6):970-7.

40. Dias EG, Souza ELS, Mishima SM. Contribuições da enfermagem na adesão ao tratamento da hipertensão arterial: uma revisão integrativa da literatura brasileira. R Epidemiol Controle Infecç. 2016;6(3):138-44.

\section{Endereço do primeiro autor:}

Aline de Sousa Falcão

Universidade Federal do Piauí - UFPI

Campus Universitário Amilcar Ferreira Sobral

BR 343, Km 3,5

CEP: 64808-605 - Floriano - PI - Brasil

E-mail: alinesousafalcao@hotmail.com

\section{Endereço para correspondência:}

Igho Leonardo do Nascimento Carvalho

Universidade Federal do Piauí - UFPI

Campus Universitário Amilcar Ferreira Sobral

BR 343, Km 3,5

CEP: 64808-605 - Floriano - PI - Brasil

E-mail: igho_leonardo@yahoo.com.br 